\title{
EDITORIAL
}

\section{Current perspectives on curative catheter ablation of atrial fibrillation}

\section{C Shah, M Haïssaguerre, P Jaïs}

Following the remarkable success of catheter based ablation techniques in curing regular supraventricular tachycardias, attention has turned to atrial fibrillation

$\mathrm{F}$ ollowing the remarkable success of catheter based ablation techniques in curing regular supraventricular tachycardias, the attention of the electrophysiological community has turned upon atrial fibrillation based on the assumption that if surgical incisions with a knife can be duplicated by point by point lesions, a catheter based technique should provide results equivalent to the Maze procedure. Considering that transmurality is an inherent accompaniment of each knife incision, that a surgical atriotomy is much narrower than anything that catheters can currently achieve, and that lesion continuity and placement is assured under direct vision in the surgical field, it is not surprising to note the difficulty encountered in reproducing surgical results by catheter based techniques.

INITIAL ATTEMPTS AT LINEAR ABLATION Two early studies attempted to intervene in both atria $^{12}$ : one in a progressive incremental fashion and the other more directly imitating the surgical Maze with a biatrial approach. The essential lessons learned from these studies (and supported by more recent ones ${ }^{3}$ ) were: that creating continuous linear lesions to duplicate surgical atriotomies was difficult; that right atrial lesions alone were safe but ineffective; that left atrial lesions improved success rates though at a significant morbidity (including proarrhythmic left atrial re-entry) and even mortality cost; but most importantly perhaps, both studies showed the feasibility of cure by catheter based techniques in patients with paroxysmal and persistent atrial fibrillation.

\section{RECOGNITION OF INITIATION FROM THE PULMONARY VEINS}

These linear ablation procedures afforded the possibility of observing shortened paroxysms of atrial fibrillation at close quarters with mapping catheters in the left atrium. Stereotyped initiations were traced in nearly all patients to sleeves of atrial myocardium encasing the ostia of one or more pulmonary veins. ${ }^{4}$ Isolated or multiple discharges culminating in paroxysms of atrial fibrillation were observed. The exact mechanism of such activity is uncertain-hence the term "discharges". Activation during the interval between the previous sinus beat and the initial venous discharge which would support a reentrant mechanism has not been documented to date and is suggestive of triggered automaticity; the consistent coupling interval (apparently coinciding with the second half of repolarisation) may also support this hypothesis. The next phase with repetitive discharges (frequently with continuous activity in the pulmonary vein) is followed by apparently random pulmonary vein activation believed to be related to wandering rotors or fleeting re-entry. An intervening or transitional phase of re-entry within or around the pulmonary vein of origin is a likely possibility particularly since short refractory periods in the pulmonary veins coexisting with long conduction times to the left atrium (at typical short coupling intervals) provide a milieu conducive to re-entry.

\section{TRIGGER BURDEN AND MAPPING}

Irrespective of the exact arrhythmia mechanism(s) involved, ablation of the initiating source (typically within a single pulmonary vein which may even be aneurysmally dilated) suffices in selected patients to eliminate short coupled atrial extrasystoles, bouts of atrial tachycardia as well as atrial fibrillation. In many of those patients, a very rapid and irregular atrial tachycardia masquerades as atrial fibrillation on the surface ECGwith an undulating baseline without discernible $\mathrm{P}$ waves because of fusion and/or fibrillatory conduction of ectopic P waves-"focal Afib". ${ }^{5}$ Ablation at the source terminates ongoing arrhythmia, testifying to its role in maintaining the arrhythmia as well.

The garden variety of patient with paroxysmal atrial fibrillation (lasting hours to days), however, has frequent involvement of 2-3 pulmonary veins as well as additional non-pulmonary vein initiating sources. The requirement for stereotypic (and non-sustained) arrhythmias from a single source to distinguish bystander sites/branches within the territory of a given vein trunk coupled with their frequently elusive appearance renders it difficult to identify each of the multiple focal sources. Therefore, though initiations from multiple PV sources can be documented by waiting long enough, by trying different provocative manoeuvres or by restudying the patient at another more opportune moment, ${ }^{46}$ a practical and expeditious solution is to systematically ablate and disconnect the myocardial sleeve of all the pulmonary veins during sinus rhythm. Of the four pulmonary veins, the right inferior is frequently difficult to map completely and perhaps therefore its arrhythmogenic potential has 
been underestimated as indicated by recent data showing nearly $33 \%$ of arrhythmogenic initiations originating from this vein.

\section{ELECTRICAL DISCONNECTION OF PULMONARY VEIN MUSCULATURE}

Anatomical considerations dictate that the more proximal the level of ablation for the pulmonary veins, the greater the extent of disconnected myocardium, but this requires more extensive ablation because of increasing diameter and myocardial coverage proximally. Electrophysiologically definable sites of preferential inputs to the veins, however, enable disconnection to be achieved at the ostia without circumferential ablation in the majority. ${ }^{7}$ In effect therefore, these preferential inputs demarcate the electrophysiological equivalent of the left atrial-pulmonary vein junction and can be considered the electrophysiologically defined ostium.

Activation of the vein can best be appreciated with some form of circumferential mapping, as opposed to longitudinal mapping typified by a multielectrode catheter placed along the length of the vein. A thin (low profile, non-traumatic) preshaped circumferential multielectrode catheter allows continuous assessment of activation around the full circumference of the vein in addition to providing a flouroscopically visible marker for the vein with a verifiable three dimensional orientation. One or two bipole(s) or electrode(s) typically show earliest activation with later and sequential spread to the rest of the venous circumference; ablation proximal to this site will delay activation in this sector with the antipodal sector (usually) now becoming the earliest activated. Ablation of this secondarily manifest input eliminates all evidence of activation distally indicating the presence of interconnections beyond the site of ablation. Two sectors of early activation separated by one of later activation suggest two distinct breakthroughs; conversely multiple near simultaneous and contiguously activated sectors suggest multiple or coalescent (broad) inputs. Disappearance of activation in a part of the circumference after proximal ablation suggests the ablation of branched or fascicle like myocardial extensions. Integration of such activation patterns at multiple levels within the vein allows appreciation of complex branching and spiral loop like patterns. Because of the cul-de-sac nature of electrical activation in the pulmonary vein, the disappearance of all distal (circumferentially recordable) potentials is a clear and unarguable indicator of conduction block.

Distinguishing target pulmonary vein potentials from far field atrial potentials is important in order to avoid unnecessary ablation which could result in vein stenosis or avoidable collateral damage (for example, to the lung or the phrenic nerve). In the right sided pulmonary veins both right as well as left atrial potentials can be identified (by mapping both sides of the interatrial septum) while in the left pulmonary veins, the nearby appendage is the most common origin of non-pulmonary vein potentials: distal coronary sinus pacing by anticipating left atrial appendage activation can distinguish the two. Exit block is frequently seen during mapping of the pulmonary veins and may simply reflect a reduced safety factor; therefore entrance block of activation into the vein from the larger current source of the left atrium is likely to reflect complete bidirectional block with greater surety. In addition, the demonstration of pulmonary vein to left atrium block during sinus rhythm is hampered by the occurrence of threshold effects when pacing from the pulmonary veins; at low outputs pulmonary vein capture may or may not occur (depending upon the proximity to pulmonary vein muscle, which is difficult to ascertain in case of a discrete fascicle) while at higher outputs direct (electrotonic) capture of the adjacent left atrial appendage and sometimes posterior left atrium or right atrium can occur, even in the complete absence of pulmonary vein potentials.
Typically circumferential mapping allows electrophysiologically guided disconnection of the four pulmonary veins to be accomplished successfully in nearly $100 \%$ of patients and quite rapidly, sometimes within one hour. In this context, the potential role of circumferential ablation devices is debatable since the gain in efficacy (if any) would be limited. Ablative energy could be unnecessarily delivered at bystander sites with resulting collateral damage, and/or may not be sufficiently concentrated or focused to ablate a discrete fascicle.

This strategy of expeditious pulmonary vein disconnection without documented proof of arrhythmogenicity can only be justified by a sufficiently low risk of side effects-notably pulmonary vein stenosis. The use of limited radiofrequency power, minimising the circumferential extent of ablation and targeting the most proximal segment (usually the largest diameter) are all important in limiting the frequency of this difficult to treat complication to $1-2 \%$ of ablated pulmonary veins. Non-occlusive stenosis limited to a single pulmonary vein (typically draining about half of one lung) usually has no significant clinical consequences.

\section{AFTER PULMONARY VEIN DISCONNECTION}

Following disconnection, provocative manoeuvres in the form of isoprenaline infusion and rapid atrial pacing are performed. Spontaneous arrhythmia (occurring after at least one sinus beat following the cessation of pacing, since the induction of atrial fibrillation during pacing is probably non-specific in terms of determining the spontaneous initiating source) at this juncture is necessarily of non-pulmonary venous origin. Such non-pulmonary vein initiating (and maintaining) focal sources are found in up to $30 \%$ of patients, in whom atrial fibrillation can be provoked even after successful disconnection of the pulmonary veins. Unlike the pulmonary veins with their relatively constant location and arbour-like structurewhich is what allows anatomic disconnection-these focal sources present specific problems. Initiation of sustained atrial fibrillation coupled with the limited electrode coverage possible through the transseptal route of left atrial access makes their localisation difficult. Most frequently located in the left atrium, they are preferentially found in the posterior left atrium and surrounding the ostia of the pulmonary veins. Less commonly, they may be found extra left atrially, but with decreasing frequency as a function of distance from the pulmonary vein ostia. They may rarely be located epicardially within the coronary sinus; within a persistent left superior vena cava draining into the coronary sinus (an abnormal adult remnant of a usually vestigial left common cardinal vein which in the normal adult is the vein or ligament of Marshall); or traced to the septum; or, in the right side of the heart, the (right) superior vena cava or in the vicinity of the crista terminalis.

Supplemental ostial ablation aimed at late or fractionated potentials may eliminate some of these recurrences; for some others, opportune mapping during periods of non-sustained (thus mappable) arrhythmia can allow successful ablation. When these non-pulmonary vein sources are multiple or trigger sustained atrial fibrillation, they are difficult to map during the few opportunities offered by early or immediate reinitiation following cardioversion, and different options may have to be tried. Better mapping techniques based on the noninvasive analysis of surface ECG P waves, multielectrode basket type catheters designed for the left atrium, or projection of intracardiac or body surface potentials to modelled endocardial boundaries (based on Laplace's equation) may allow localisation and elimination by wide or even anatomical ablation of these non-pulmonary vein sources. Adjuvant linear ablation-a necessary component for ablation of chronic atrial fibrillation-can also terminate or reduce the duration of paroxysms and facilitate mapping; though at the cost of possible 
left atrial proarrhythmia (in case of incomplete lines) and perhaps a variable effect on left atrial contractile function. Previously ineffective antiarrhythmic drug treatment can eliminate residual atrial fibrillation in about $30-40 \%$ of these patients.

In conclusion, electrical disconnection of pulmonary veins is easily demonstrable and routinely possible with a low risk of pulmonary vein stenosis, which compares favourably with the risk of persistent atrial fibrillation, and antiarrhythmic and anticoagulant drug treatment. Demonstration of elimination of atrial fibrillation (which is particularly easy in patients with frequent paroxysms) frees the patient not only from antiarrhythmic treatment but also from oral anticoagulation. This procedure though does not eliminate paroxysmal atrial fibrillation in about $30 \%$ of patients who typically have longer duration of atrial fibrillation and non-pulmonary focal sources. Thus, at present, resistance to antiarrhythmic drugs should determine when this therapeutic option should be offered to a specific patient with paroxysmal atrial fibrillation. With future reduction in the risk profile of the procedure and more complete elimination of non-pulmonary venous foci and/or more efficient and safe deployment of linear lesions, the majority of patients with paroxysmal atrial fibrillation could become amenable to catheter based curative treatment.

\section{Authors' aaffiliations}

D C Shah, $M$ Haïssaguerre, P Jaïs, Hôpital Cardiologique du Haut-Lévêque, Avenue de Magellan, 33604 Pessac Cedex, France

\section{REFERENCES}

1 Haïssaguerre $M$, Jaïs $P$, Shah DC, et al. Right and left atrial radiofrequency catheter therapy of paroxysmal atrial fibrillation. $J$ Cardiovasc Electrophysiol 1996;7: 1132-44.

2 Swartz JF, Pellersels G, Silvers J, et al. A catheter-based curative approach to atrial fibrillation in humans [abstract]. Circulation 1994;90(pt II): $1-335$

3 Ernst S, Shluter M, Ouyang F, et al. Modification of the substrate for maintenance of idiopathic human atrial fibrillation: efficacy of radiofrequency ablation using nonfluoroscopic catheter guidance. Circulation 1999:100:2085-92.

4 Haïssaguerre $M$, Jaïs $P$, Shah DC, et al. Spontaneous initiation of atrial fibrillation by ectopic beats originating in the pulmonary veins. N Engl J Med 1998;339:659-66.

5 Jaïs $\mathbf{P}$, Haïssaguerre $M$, Shah DC, et al. A focal source of atrial fibrillation treated by discrete radiofrequency ablation. Circulation 1997;95:572-6.

6 Haïssaguerre $M$, Jaïs $P$, Shah DC, et al. Electrophysiological end point for catheter ablation of atrial fibrillation initiated from multiple pulmonary venous foci. Circulation 2000;101:1409-17.

7 Haïssaguerre $M$, Shah DC, Jaïs $P$, et al. Electrophysiological breakthroughs from the left atrium to the pulmonary veins. Circulation 2000;102:2463-5

\section{IMAGES IN CARDIOLOGY}
Mechanism of transient exercise induced ST segment elevation in non- $Q$ wave lead: a scintigraphic co-relation

A

67 year old man underwent ${ }^{99 \mathrm{~m}} \mathrm{Tc}$-sestamibi single photon emission computed tomography (SPECT) for evaluation of atypical epigastric pain. His baseline 12 lead ECG revealed anterior ST elevation $(1.5 \mathrm{~mm})$ at rest without Q waves. During exercise SPECT imaging the patient developed ST segment elevation over leads V2-V4 (7 mm in V3) at 4.2 minutes into a Bruce protocol. The ST segment changes persisted for two minutes. Stress (S) and rest (R) studies were performed on separate days.

The figure shows a "bulls-eye" plot of myocardial perfusion and wall thickening (derived from gated images). The anteroseptal left ventricular (LV) wall showed $>90 \%$ reduction of perfusion following stress, suggesting transmurality of the ischaemic region. This was associated with a large fall in left ventricular ejection fraction (EF) (from 67\% to 47\%) and gross increase in left ventricular volumes even one hour after stress (when images were acquired). Persistent wall thickening abnormality was present at one hour after stress suggesting post-ischaemic stunning. Normalisation of perfusion was associated with improvement in wall thickening and $\mathrm{LV}$ function at rest (on a separate day). Coronary angiography revealed $>90 \%$ lesions in proximal and dominant left anterior descending artery, with diffuse right coronary artery disease $(<50 \%)$.

Gated SPECT clearly demonstrates the value of simultaneous assessment of perfusion and function, which reflects the functional significance of the myocardial ischaemia. The mechanism of transient ST segment elevation is likely to be the result of transmural ischaemia leading to severe haemodynamic derangement caused by myocardial stunning.
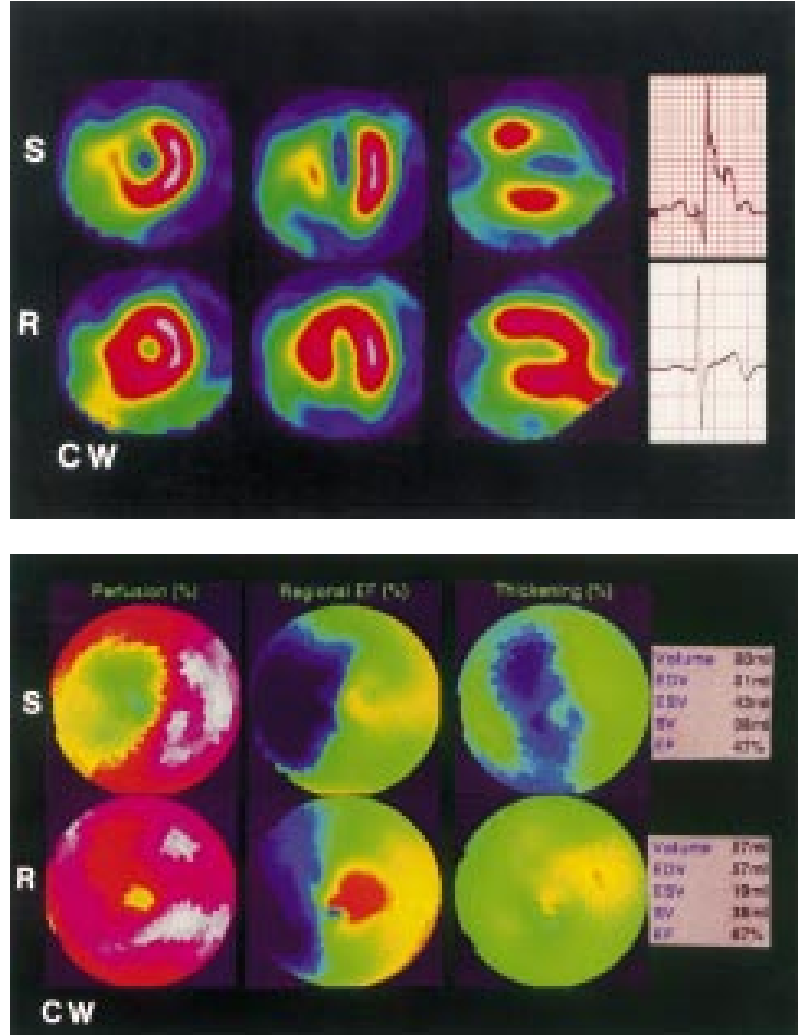